\title{
CURSO NATURAL. DA ELIMINAÇÃO DE OVOS DE Eurytrema sp LOSS, 1907 NAS FEZES DE BEZERROS
}

\author{
THE ELIMINATION PATTERN OF EGGS OF Eurytrema sp LOSS, 1907 IN THE FAECES OF TAURINE AND \\ ZEBUINE CROSSBRED CALVES
}

Jackson Victor de ARAÚJO' ; Pacífico Antonio Diniz BEL.ÉM²

\begin{abstract}
RESUMO
Treze bezerros 3/4 holandês x 1/4 zebu, nascidos em diferentes estaçōes do ano de 1989 e criados intensivamente, foram transferidos para pastagens de capim gordura (Melinis minutiflora) entre 7 meses e 1 ano de idade, no final do período chuvoso (março de 1990), a fim de ser acompanhado o curso natural da eliminação de ovos de Eurytrema sp por grama de fezes (OPG). De $15 \mathrm{em} 15$ dias, foram feitas as colheitas de fezes para obter as contagens de OPG durante 18 meses. Os primeiros animais que apresentaram exames positivos (a partir de agosto de 1990) foram os que tiveram as maiores contagens de OPG durante o período experimental. Os demais apresentaram os primeiros exames positivos em diferentes ocasiōes e as contagens de OPG destes foram baixas e oscilantes. Três animais apresentaram exames negativos. Provavelmente, a maioria dos bovinos criados em regime de pastejo desta região deverão apresentar infecção parasitária por Eurytrema $\mathrm{sp} \mathrm{cm}$ alguma fase de suas vidas.
\end{abstract}

UNITERMOS: Eurytrema: Fezes; Bezerros

\section{INTRODUÇÃO}

Segundo COSTA et al. ${ }^{8}$ ( 1986 ) o diagnóstico dá infecção por Eurytrema sp em bovinos, no Brasil, tem sido registrado por vários autores.

Informações disponiveis na literatura revelam que a infecção por Eurytrema sp é mais freqüentemente constatada $\mathrm{cm}$ bovinos adultos, sobretudo os mais velhos ${ }^{4}$, embora bezerros também possam ser acometidos 3,6,7,10.11,15,16.

Este trabalho teve o objetivo de acompanhar o curso natural da eliminação de ovos de Eurytrema sp por grama de fezcs de bovinos introduzidos ao regime de pastejo no final da estação chuvosa.

\section{MATERIAL E MÉTODO}

O experimento foi conduzido no município de Viçosa, Estado de Minas Gerais - Brasil, com latitude de $20^{\circ} 45^{\circ} 20^{\prime \prime} \mathrm{S}$, longitude de $42^{\circ} 52^{\prime} 40^{\prime \prime} \mathrm{W}$ e altitude de $649 \mathrm{~m}$.

Treze bezerros 3/4 holandês e 1/4 zebu ( 4 machos e 9 fêmeas), nascidos em diferentes estações do ano de 1989, foram criados em baias individuais até os 2 meses de idade. Cada animal recebia, diariamente, $4 \mathrm{~kg}$ de leite "in natura" e $2 \mathrm{~kg}$ de concentrado com $20 \%$ de proteína bruta e $70 \%$ de nutrientes digestíveis totais, além de sal mincral, água e capim "elefante" picado (Pinnisetum purpureum) à vontade. Dos dois aos quatro meses de idade os bezerros foram transferidos para bezerreiros coletivos, reduzindo-se o leite fornecido para $2 \mathrm{~kg}$, sendo o mesmo, após 4 meses de idade, completamente retirado, porém mantendo-se o restante da alimentação.

Entre 7 meses e 1 ano de idade, no final do período chuvoso (março de 1990), os bezerros foram transferidos para pastagens de capim gordura (Melinis minutiflora), com taxa de lotação de 0,60 animal/ha, onde eram oferecidos água e sal mineral à vontade e, diariamente para cada animal, $0,5 \mathrm{~kg}$ da ração acima descrita.

Por um periodo de 18 meses, de $15 \mathrm{em} 15$ dias, foram feitas as colheitas de fezes, diretamente do reto de cada animal, que foram utilizadas para realizar os exames das contagens de ovos de Eurytrema sp por grama de fezes (OPG). Foi empregada técnica modificada de DENNIS et al. ${ }^{9}$ (1954), segundo BELÉM et al. ${ }^{2}$ (1992).

Dados meteorológicos foram obtidos em estação especializada na própria fazenda onde o experimento foi conduzido. 


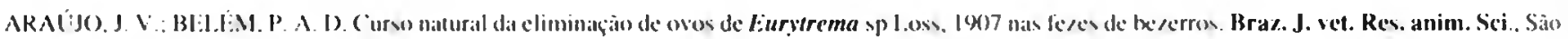
P:ulk, . 31, 11.2. P. 125.4. 1944

\section{RESLLTADOS E DISCUSSÃo}

As contagens de ovos de Eurytrema sp por grama de fezes (OP(i) (Tab.1) mostram que os primeiros animais que apresentaram exames positivos foram os de maiores contagens de OPG c somente três animais apresentaram exames negativos durante o período experimental. Os demais, após apresentarem us primeiros exames positivos. exibiram contagens baixas ou exames negativos de ovos nas lezes. Feten resultados indicimn que be cerros também podem ser parasilados, mas com o passar dat idade. mais animais do rebanho vão se infectando e. provavelmente. a maioria dos animais desta regiano deverá apresentar infecção parasitária por Euryerema sp em alguma fase de suas vidas.

Quanto às oscilaçóes, ausências e valores baixos de OPG. SAKAMOTO el al. ${ }^{1+}(1980)$, ao trabalharem com bovinos naturalmente infectados è utilizando o método de sedimentação. reconheceram ser difícil a deleç̧ão de ovos de Eurytrema coelomaticum. Ainda segundo SAKAMOTO el al. ${ }^{13}(1980)$. 1) valor do OPG em bovinos parasitados por Eurytrema coelomaticum apresenta oscilaçóes diárias e. algumbas vezes. ocorrem casos de resulados negativos de ovos nas lezes. BBELLE' (1991), ao aplicar modelos matemáticos, relata que a probabilidade de encontrar valores elevados de OPG é remota. Além disso, MARTIN'2 (1972): (HIMONE:
ITAGAKE.5 (1976) e BH:I.EM' (1991) observaram. em seus trabalhos, que quando a carga parasitária é muito baixa a postura de ovos também o é. o que dificulta o diagnóstico coproparasitológico da infecção por Eurytrema sp.

Os animais E. L. M $N$ apresentaram as contagens de OPG mais elevadas e raramente apresentaram resultados negativos, interferindo grandemente nos valores médios das contagens de OPG entre os animais positivos (fig. 1). Bsta ligura tannbém mostra que o valor médio mais elevado (1.3.5 ()PG) ocorreu na segunda quinzena de abril de 1991. Eim geral. os valores apresentaram em um platô quase que constante a partir de outubro de 1990). Pode-se supor que estes animais. provavelmente. desenvolveram pouca resposla imunitária ao parasia.

Na lïg.2 demonstra-se gue as temperaturas pouco oscilaram. mas a precipitação plusial delimitou o clima em estação chuvosa (outubro a março) e estaçăo seca (abril a setembro).

\section{CONCLUSÃo}

A maioria dos animais adquiriu, naturalmente, a infeç̧äo parasitária por Eurytrema sp durante o periodo experimental. o que sugere a importancia desta helmintose para os bovinos na regiăo de Viçona.

\section{SUMMARY}

Thirten worm frec crossbred calves (3/4 Holstein $\times 1 / 4$ Zebu) aging from 7 to 12 months were put in a pasture of . Melinis minutiflora at the end of the rainy season (March. 1990). Starting from August. 1990), during 18 months, at every 15 days. a faccal sample from each calf wats colleced for Eurytrema egg counts. The first animals which eggs were found in their laeces were also the ones which reached the highest faecal egg counts. The remaining calves presented lower EP(i counts and in threc of them negative counts were obtained from time to lime.

LNITH.MS: Eurylrema: laseces: Calves

\section{REFERÊNCIAS BIBIJIOGRÁFICAS}

(0)-BLILAM. P.A.D. Aspectos ligados ao diagnóstico da infecção por Eurytrema sp em bovinos. Botucatu, 1901. Tese (Doutorado) - Faculdade de Medicina Veterinária e \%ootecnia. Iniversidade Fstadual Paulistat. Compus de Botucatu.

02-BI:L.ÍM.P.A.D.: OL.IVEIRA. M.R.O.:PADOVANI, C.R. Adaptação dal lécnica de Demnis. Stone \& Swanson para diagnóstico copro-parasitológico de infecção naltural por Eurytrema sp em bovinos. Braz. J. vet. Res. anim. Sci. São Paulo. v.29. n.2. p.303-7. 1992.
0.3-BELLATO. V.: SOUZA. A.P.: RAMOS. C.I.; SA.NTOS. A.A. Eurytrema sp em bovinos no município de Lages. In: CICLO IE ATLALIZAÇÃO EM MEDICINA VFTHRINÁRIA. 2.. Lages. 1981 .Anais. Lages. UHSC, 1981 . p.207.

04-CARVAI.HO. J.C..M. Contribuição para o conhecimento da fauma helmintologica de Minas Gerais. Ceres. v.l. 11.5. p.411-23, 194().

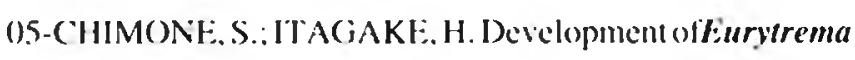


A RAÚJO, J. V.; BELl.́.M. P. A. D. Curso natural da eliminação de ovos de Eurytrema sp Loss, 1907 nas fezes de bezerros. Braz. J. vet. Res. anim. Sci.. São Paulo. v. 31, n.2, p. 125-9, 1994.

pancreaticum (TREMATOI)A). II. Development in definitive horts. Bull. Azahu. Vet. College. v. I. n.2, p. $73-81,1976$.

06-COSTA, A.J.; CAMPOS. M.S.; KASAI. N.; PAULILLLO, A.C.; COSTA. A.F. Estudo preliminar sobre helmintoses gastrointestinais de bovinos no município de Jaboticabal, Estado de São Paulo, Brasil. Científica, v.6, n.3, p.443-9, 1978.

07-COSTA, H.M.A.; FREITAS, M.G.; GUIMARĀES, M.P. Prevalência e intensidade de infestação por helmintos de bovinos procedentes da área de Três Coraçōes. Arq. Esc. Sup. Vet. Univ. Rural Est. M. Gerais, v.22, p.95-101, 1970.

08-COSTA. H.M.A.; GUIMARÃES, M.P.; LEITE. A.C.R.; LIMA, W.S. Distribuição de helmintos parasitos de animais domésticos no Brasil. Arq. Bras. Med. Vet. Zootec., v.38, n.4, p.465-579, 1986.

09-DENNIS, W.R.: STONE, W.M.; SWANSON, L.E. A new laboratory and ficld diagnostic test for fluke ova en faeces. J. Amer. Vet. Med. Ass., v.134, p.47-50, 1954.

10-HORTA, P.P. Distomatose pancreática e glicosúria em bovinos. A Lavoura, v.22, n.3/4, p.157-8, 1918.

11-KONO. I.; FUKUGOSHI. S. Studies on the existence of eggs of pancreas fluke in the wall of the pancreatic duct and its significance. Bull. Fac. Agric. Kagoshima Univ., v.12. p.197-206, 1966.
12-MARTIN, O.C. The incidence of Eurytrema pancreaticum (Loss, 1907) in dairy cattle at the DTRJ Farm. I'hillipp. Agric., v.56, n. 1/2, p.25-34, 1972.

13-SAKAMOTO, T.; KONO, I.; YASUDA, N.; YAMAMOTO. Y.; NAKAGAWA, H. Studies on Eurytrema coelomaticum. II. The anthelmintic efficiency of nitroxynil and praziquantel against Eurytrema coelomaticum in cattle. Mem. Fac. Agric. Kagoshima Univ., v. 16, p.93-101, 1980.

14-SAKAMOTO, T.; TASHIRO, T.; WATANABE, S.; SAKAMOTO, T.; KONO, I.; YASUDA. N. Clinicopathological findings of cattle infected with Eurytrema coelomaticum. Bull Fac. Agric. Kagoshima Univ., v.30, p.117-22, 1980 .

15-TORRES, C.M.; PINTO, C. Processos patogênicos determinados pelos trematodas Eurytrema fastosum c E.coelomaticum (DICROCOELLIDAE). Mem. Inst. Oswaldo Cruz, v.31, n.3, p.731-46, 1936.

16-VIANA, S.S.S. Técnica coproscópica de sedimentação para concentração de ovos de Eurytrema sp Loss, 1907. São Paulo, 1985. Tese (Doutorado) - Instituto de Ciências Biomédicas, Universidade de São Paulo.

Recebido para publicação em (05/04/93 Aprovado para publicação em 19/10/93 
ARAUJO, J. V.: BLLLÉM. P. A. D. Curso natural da eliminação de ovos de Eurytrema sp Loss. 1907 nas fezes de bezertos. Hraz. J. vet. Res. anim. Sci., São Paulo. v. 31. ก.2. p. 125-9. 1994

TABEIA 1

Conlagens quinzenais de ovos de Eury/rema sp por grama de fezes (OPG) dos bovinos durante o período de março de 1990 a agosto de 1991 . Viçosa - MG.

\begin{tabular}{|c|c|c|c|c|c|c|c|c|c|c|c|c|c|c|c|c|c|c|c|c|c|c|c|c|c|c|c|c|c|c|c|c|c|c|c|c|c|c|}
\hline \multirow[t]{3}{*}{ Animai } & \multirow[t]{3}{*}{ Sexo } & \multirow{3}{*}{$\begin{array}{c}\text { Mês/Ano } \\
\text { de } \\
\text { Nascimento }\end{array}$} & \multicolumn{36}{|c|}{ OPG QUINZENAL DE Eurytrema sp } \\
\hline & & & \multicolumn{2}{|c|}{$M$} & \multicolumn{2}{|c|}{ A } & \multicolumn{2}{|c|}{$\mathbf{M}$} & \multicolumn{2}{|c|}{ 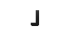 } & \multicolumn{2}{|c|}{ J } & \multicolumn{2}{|c|}{ A } & \multicolumn{2}{|c|}{5} & \multicolumn{2}{|c|}{0} & \multicolumn{2}{|c|}{$\mathbf{N}$} & \multicolumn{2}{|c|}{ D } & \multicolumn{2}{|c|}{ J } & \multicolumn{2}{|c|}{$\mathbf{F}$} & \multicolumn{2}{|c|}{$M$} & \multicolumn{2}{|c|}{ A } & \multicolumn{2}{|c|}{$\mathbf{M}$} & \multicolumn{2}{|c|}{$\mathrm{J}$} & \multicolumn{2}{|c|}{$\mathrm{J}$} & \multicolumn{2}{|c|}{ A } \\
\hline & & & $x$ & $\mathbf{Y}$ & $x$ & $Y$ & $x$ & $Y$ & $x$ & $Y$ & $x$ & $Y$ & $x$ & $Y$ & $x$ & $Y$ & $x$ & $Y$ & $x$ & $Y$ & $x$ & $\mathbf{Y}$ & $x$ & $\mathbf{Y}$ & $x$ & $Y$ & $x$ & $Y$ & $x$ & $\mathbf{Y}$ & $x$ & $Y$ & $x$ & $Y$ & $x$ & $Y$ & $x$ & Y \\
\hline A & $\mathbf{M}$ & $08 / 89$ & 0 & 0 & 0 & 0 & 0 & 0 & D & 0 & 0 & 0 & 0 & 0 & 0 & 0 & 0 & 0 & 0 & 0 & 0 & 0 & 0 & 0 & 0 & 0 & 0 & 0 & 0 & 0 & 0 & 1 & 0 & 0 & 0 & 0 & 0 & 0 \\
\hline B & $\mathbf{F}$ & $07 / 89$ & 0 & 0 & 0 & 0 & 0 & 0 & 0 & 0 & 0 & 0 & 0 & 0 & 0 & 0 & 0 & 0 & 0 & 0 & 0 & 0 & 0 & 0 & 0 & 0 & D & 0 & 0 & 0 & 1 & 0 & 0 & 0 & 0 & 0 & 0 & 0 \\
\hline C & $\mathbf{F}$ & $07 / 89$ & 0 & 0 & 0 & 0 & 0 & 0 & 0 & 0 & 0 & 0 & 0 & 0 & 0 & 0 & 0 & 0 & 0 & 0 & 0 & 0 & 0 & 0 & 0 & 1 & 0 & 0 & 0 & 0 & 0 & 1 & 0 & 0 & 0 & 0 & 1 & 0 \\
\hline D & $\mathbf{F}$ & $07 / 89$ & 0 & 0 & 0 & 0 & 0 & 0 & 0 & 0 & 0 & 0 & 0 & 0 & 0 & 0 & 0 & 0 & 1 & 2 & 0 & 0 & 0 & 0 & 0 & 0 & 0 & 0 & 1 & 0 & 1 & 0 & 0 & 0 & 0 & 1 & 0 & 1 \\
\hline E & $M$ & $06 / 89$ & 0 & 0 & 0 & 0 & 0 & 0 & 0 & 0 & 0 & 0 & 1 & 0 & 0 & 0 & 0 & 0 & 3 & 2 & 10 & 0 & 2 & 0 & 0 & 2 & 0 & 3 & 4 & 8 & 7 & 4 & 4 & 0 & 1 & 0 & 2 & 3 \\
\hline $\mathbf{F}$ & $\mathbf{F}$ & $06 / 89$ & 0 & 0 & 0 & 0 & 0 & 0 & 0 & 0 & 0 & 0 & 0 & 0 & 0 & 0 & 0 & 0 & 0 & 0 & 0 & 0 & 0 & 0 & 0 & 0 & 0 & 0 & 0 & 0 & 0 & 0 & 0 & 0 & 0 & 0 & 0 & 0 \\
\hline $\mathbf{G}$ & $\mathbf{F}$ & $06 / 89$ & 0 & 0 & 0 & 0 & 0 & 0 & 0 & 0 & 0 & 0 & 0 & 0 & 0 & 0 & 0 & 0 & 0 & 0 & 0 & 0 & 0 & 0 & 0 & 0 & 0 & 0 & 0 & 0 & 0 & 0 & 0 & 0 & 0 & 0 & 0 & 0 \\
\hline H & $\mathbf{F}$ & $06 / 89$ & 0 & 0 & 0 & 0 & 0 & 0 & 0 & 0 & 0 & 0 & 0 & 0 & 0 & 0 & 0 & 0 & 0 & 0 & 0 & 0 & 0 & 0 & 0 & 0 & 0 & 0 & 0 & 0 & 0 & 0 & 0 & 0 & 0 & 0 & 0 & 0 \\
\hline 1 & $\mathbf{M}$ & $06 / 89$ & 0 & 0 & 0 & 0 & 0 & 0 & 0 & 0 & 0 & 0 & 0 & 0 & 0 & 0 & 0 & 0 & 0 & 0 & 0 & 0 & 0 & 0 & 0 & 0 & 0 & 0 & 0 & 0 & 0 & 2 & 0 & 0 & 0 & 0 & 0 & 0 \\
\hline J & $F$ & $05 / 89$ & 0 & 0 & 0 & 0 & 0 & 0 & 0 & 0 & 0 & 0 & 0 & 0 & 0 & 0 & 0 & 0 & 0 & 0 & 0 & 0 & 0 & 0 & 5 & 1 & 0 & 1 & 0 & 0 & 1 & 0 & 0 & 2 & 0 & 0 & 0 & 0 \\
\hline $\mathbf{L}$ & $\mathbf{M}$ & $05 / 89$ & 0 & 0 & 0 & 0 & 0 & 0 & 0 & 0 & 0 & 0 & 0 & 9 & 10 & 1 & 1 & 6 & 10 & 11 & 13 & 8 & 5 & 4 & 4 & 24 & 1 & 9 & 6 & 24 & 12 & 23 & 6 & 11 & 9 & 4 & 12 & 9 \\
\hline $\mathbf{M}$ & $F$ & $03 / 89$ & 0 & 0 & 0 & 0 & 0 & 0 & 0 & 0 & 0 & 0 & 0 & 0 & 0 & 0 & 0 & 0 & 1 & 2 & 3 & 2 & 3 & 0 & 1 & 6 & 0 & 3 & 0 & 7 & 5 & 1 & 0 & 8 & 5 & 4 & 5 & 5 \\
\hline $\mathbf{N}$ & $F$ & $02 / 89$ & 0 & 0 & 0 & 0 & 0 & 0 & 0 & 0 & 0 & 0 & 0 & 0 & 1 & 1 & 0 & 0 & 3 & 2 & 2 & 4 & 0 & 0 & 3 & 6 & 1 & 9 & 7 & 15 & 14 & 5 & 0 & 7 & 8 & 8 & 4 & 8 \\
\hline
\end{tabular}

$\mathrm{M}=$ Sexo masculino

$\mathrm{F}=$ Sexo feminino

$\mathrm{X}=\mathrm{I}^{2}$ quinzena

$\mathrm{Y}=2^{x}$ quinzena

$\mathrm{M} \ldots \mathrm{A}=$ meses do ano

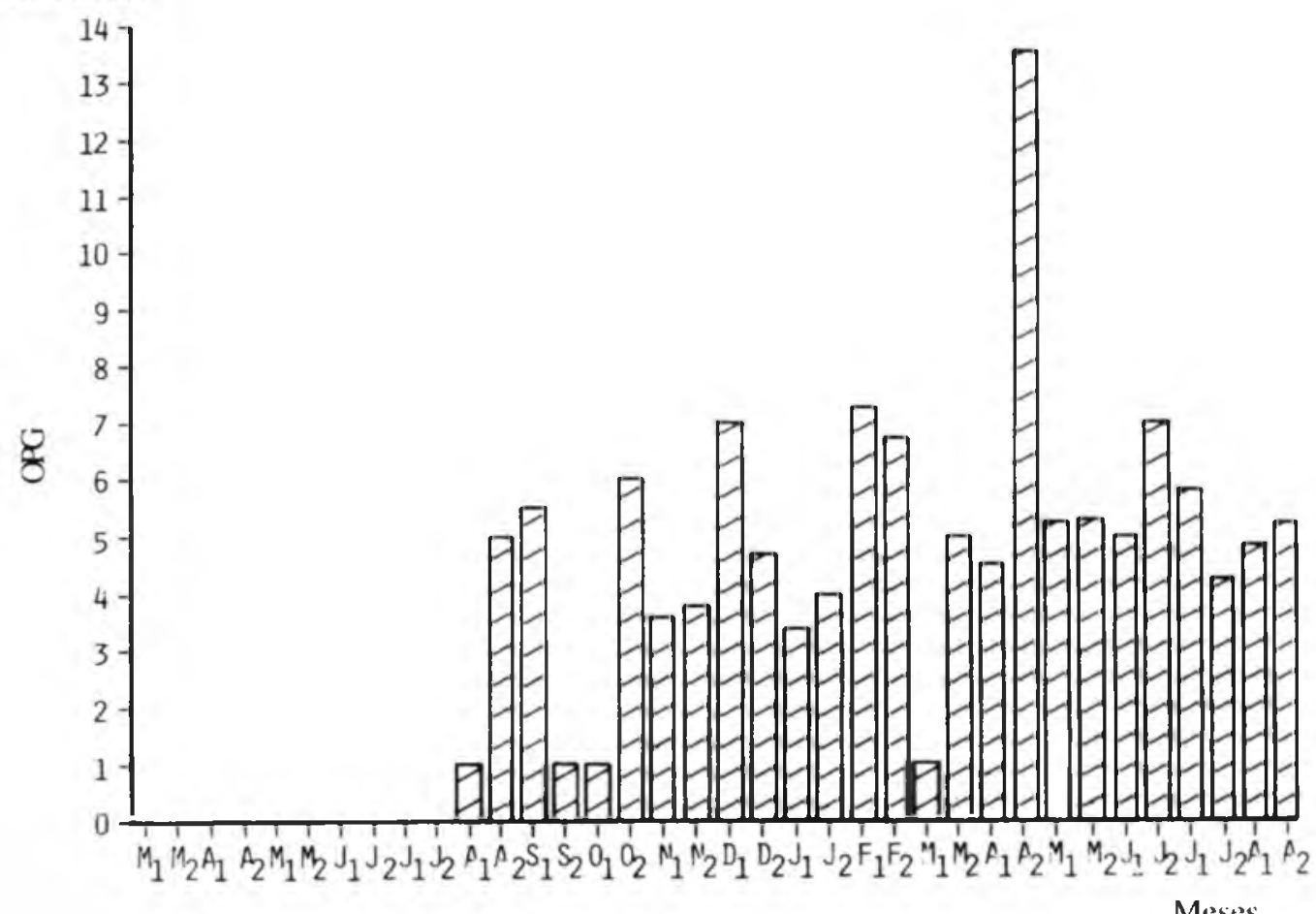

FIGURA 1

Meses

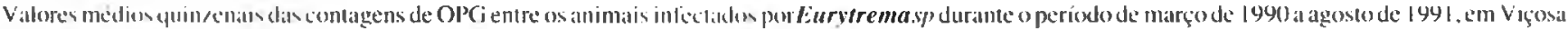



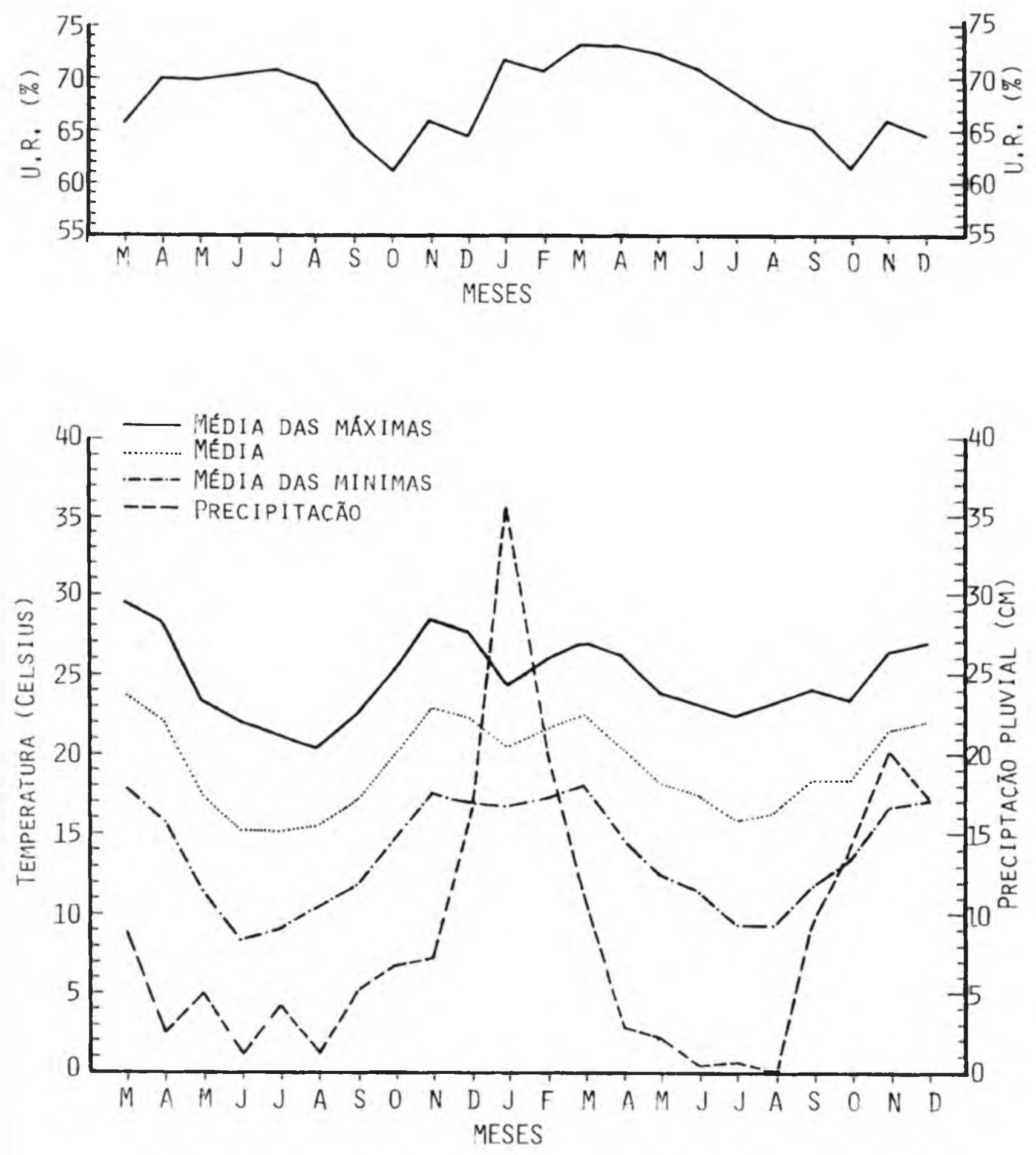

FIC;LRA 2

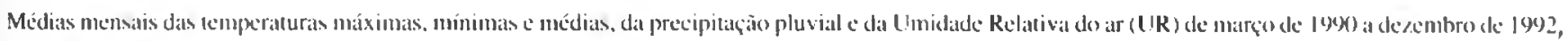
enn Viçosia - MG. 